\title{
High prevalence of diabetes mellitus and metabolic syndrome in a South African coloured population: Baseline data of a study in Bellville, Cape Town
}

\author{
Rajiv T Erasmus, David J Soita, Mogamat S Hassan, Ernesto Blanco-Blanco, Zelda Vergotine, Andre P Kengne, Tandi E Matsha
}

Objective. The coloured population has the second-highest prevalence of diabetes in South Africa. However, the data were based on a study conducted almost 20 years ago in a peri-urban coloured population of the Western Cape. We aimed to determine the prevalence of diabetes mellitus and metabolic syndrome in an urban coloured population in South Africa.

Design. In a cross-sectional survey, 642 participants aged $\geq 31$ years were drawn from an urban community of Bellville South, Cape Town, from mid-January 2008 to March 2009. Type 2 diabetes was assessed according to the WHO criteria, and metabolic syndrome was based on the International Diabetes Federation (IDF), ATP III and 2009 Joint Interim Statement (JIS) definition.

Results. The crude prevalence of $28.2 \%$ (age-adjusted $26.3 \%$, 95\% confidence interval (CI) 22.0 - 30.3) for type 2 diabetes was: $4.4 \%$ (age-adjusted $3.2 \%, 95 \%$ CI 1.6 - 4.9) for impaired fasting glycaemia, and $15.3 \%$ (age-adjusted 15.0\%, 95\% CI 11.4 - 18.6) for impaired glucose tolerance. Undiagnosed type 2 diabetes was present in $18.1 \%$ (age-adjusted $16.8 \%$, 95\% CI 13.3 - 20.4). The crude prevalence of metabolic syndrome was higher with the JIS definition (62.0\%) than the IDF (60.6\%), and the National Cholesterol Education Program (NCEP) ATP III (55.4\%). There was good overall agreement between the MetS criteria, $\mathrm{k}=0.89$ (95\% CI 0.85 - 0.92).

Conclusion. The prevalence of diabetes has increased hugely in the coloured community, and the high prevalence of undiagnosed diabetes portends that cardiovascular diseases might grow to epidemic proportions in the near future in South Africa.

S Afr Med J 2012;102(11):841-844. DOI:10.7196/SAMJ.5670
The South African coloured population group comprises people whose ancestry is about $32-43 \%$ Khoisan, $20-36 \%$ black, $21-28 \%$ white and $9-11 \%$ Asian. ${ }^{1}$ This population has the second-highest prevalence of diabetes in South Africa, after that of the Indian population..$^{2-4}$ However, this finding was based on a study almost 20 years ago in a peri-urban coloured population of the Western Cape. ${ }^{2}$ Since then, South Africa has undergone significant political changes that have been accompanied by rapid urbanisation and economic changes, particularly among the coloured and other non-white population groups. These epidemiological changes are often paralleled by increases in lifestyle diseases such as dysglycaemia, obesity and high blood pressure that are associated with the metabolic syndrome (MetS).

Division of Chemical Pathology, Faculty of Health Sciences, National Health Laboratory Service (NHLS) and Stellenbosch University

Rajiv T Erasmus, FCPath

Mogamat S Hassan, MSc

Zelda Vergotine, MSc

Faculty of Health and Wellness Science, Cape Peninsula University of Technology, Cape Town

David J Soita, MTech

Mogamat S Hassan, MSc

Zelda Vergotine, MSc

Tandi E Matsha, PhD

Department of Chemical Pathology, Walter Sisulu University, Mthatha, Eastern Cape Ernesto Blanco-Blanco, FCPath

National Collaborative Research Programme for Cardiovascular and Metabolic Diseases, South African Medical Research Council, Cape Town

Andre P Kengne, MD, PhD
MetS is common in subjects with type 2 diabetes, and is associated with micro- and macrovascular complications. ${ }^{5}$ In developing countries, type 2 diabetes is often undiagnosed, and related cardiovascular disease (CVD) risk may therefore far exceed that of known cases owing to the unmanaged glycaemic state and other risk factors. ${ }^{6}$ Studies of MetS in Africa and elsewhere have been hampered by differences in major sets of criteria for defining MetS. ${ }^{8}$ Recently, however, the International Diabetes Federation (IDF) and the American Heart Association/ National Heart, Lung and Blood Institute (AHA/NHLBI) released a consensus definition for MetS (the Joint Interim Statement (JIS) in which abdominal obesity was removed as a prerequisite for the diagnosis of metabolic syndrome. ${ }^{9}$ Consequently, the JIS definition classifies an individual as having MetS if 3 of the following criteria are met: raised blood pressure, elevated triglycerides, low high-density lipoprotein cholesterol levels, central obesity, and hyperglycaemia.

The last epidemiological report from South Africa on the prevalence of diabetes in the coloured population was 15 years ago, using the 1985 WHO criteria. ${ }^{2}$ We set out to establish the prevalence of diabetes, undiagnosed diabetes, and MetS in a coloured population residing in an urban suburb of Cape Town.

\section{Methods}

The study was approved by the Faculty of Health and Wellness Sciences ethics committee of the Cape Peninsula University of Technology, and was conducted according to the Code of Ethics of the World Medical Association (Declaration of Helsinki). Participants signed written informed consent after the procedures had been fully explained in the language of their choice. Permission was also sought from other relevant authorities to operate in the community and also to make use of designated places such as community halls and nearby schools for data and samples collection.

Research setting, research design and study population A detailed description of the research setting has been described. ${ }^{6,10}$ Bellville South is a predominantly coloured-populated area in the 
northern suburbs of Cape Town, in the Tygerberg sub-district. Our target population was subjects $\geq 31$ years old. Using a map of Bellville South, random sampling was approached as follows: the area was divided into 6 strata; within each stratum the streets were classified as short ( $\leq 22$ houses), medium ( 23 - 40 houses) and long ( $\geq 40$ houses), based on the number of houses. Two of each street type were randomly selected from each stratum. Where the number of houses was too few, a short or a medium street was randomly selected and added to the stratum. The result was a total of 16 short streets representing approximately 190 houses, 15 medium (approximately 410 houses) and 12 long streets (approximately 400 houses). From the selected streets, all household members meeting the selection criteria were invited to participate. Of subjects who met the criteria, 1000 were approached and 642 participated. The data were collected between mid-January 2008 and March 2009.

\section{Data collection}

A detailed protocol describing data collection procedures (questionnaires and physical examination) was developed. Team members comprising professional nurses and the recruitment team were trained, and a pilot study in a neighbouring community with similar demographics was performed to validate the questionnaire and to synergise the workflow. A supervisor for each team monitored their performance and was responsible for calibrating equipment according to a standard protocol. A weekly meeting was also held to assess progress, solve problems and re-train the research team. A questionnaire designed to retrospectively obtain information on lifestyle factors such as smoking and alcohol consumption, physical activity, diet, family history of CVD and diabetes mellitus, demographics, etc. was administered by trained personnel. The questionnaire was adapted from standard and recognised sources, ${ }^{11,12}$ and was pretested in a neighbouring community with similar demographics. A detailed drug history was also obtained by interrogation and by examining the clinic cards and the record of drugs that participants brought to the study site.

Clinical measurements included height, weight, hip and waist circumferences and blood pressure. Measurements were carried out by qualified healthcare professionals who underwent training to standardise all measurements before commencing the study. Blood pressure measurements were performed according to WHO guidelines. ${ }^{13}$ Weight was determined on a calibrated and standardised Sunbeam EB710 digital bathroom scale. Weight measurements were recorded to the nearest $0.1 \mathrm{~kg}$ and taken with each subject in light clothing, without shoes and socks. Height was recorded in centimetres to one decimal place using a stadiometer. Body mass index (BMI) was calculated as weight per square meter $\left(\mathrm{kg} / \mathrm{m}^{2}\right)$. Using a non-elastic tape, waist circumference was measured at the narrowest part of the torso as seen from the anterior view and hip circumference around the widest segment of the buttocks. All anthropometric measurements were performed 3 times, and the average measurements were used for analysis.

All participants except the self-reported type 2 diabetes subjects (confirmed by either medical card record or drugs in use) underwent a $75 \mathrm{~g}$ oral glucose tolerance test (OGTT) as prescribed by the WHO, with fasting blood glucose. Categories of glucose tolerance were defined applying the $1998 \mathrm{WHO}$ criteria. ${ }^{14}$ Blood samples were transported daily in an ice-pack box for processing at the Metropolis Private Pathology Laboratory (Century City, Cape Town). Plasma glucose was measured by enzymatic hexokinase method (Cobas 6000, Roche Diagnostics). Glycosylated haemoglobin $\left(\mathrm{HbA}_{1 \mathrm{c}}\right)$ was assessed by turbidimetric inhibition immunoassay (Cobas 6000, Roche Diagnostics). This method is National Glycohaemoglobin
Standardisation Programme (NGSP) certified according to Roche Diagnostics. High-density lipoprotein cholesterol (HDL-c) and triglycerides (TG) were estimated by enzymatic colorimetric methods (Cobas 6000, Roche Diagnostics). Low-density lipoprotein cholesterol (LDL-c) was calculated using Friedewald's formula. Serum cotinine was measured by chemiluminescent assay (Immulite 1000, Siemens). The JIS, ${ }^{9}$ IDF $^{8}$ and ATP III ${ }^{8}$ statements were used to define metabolic syndrome.

\section{Statistical analysis}

Statistical analysis of the data was performed using the statistical package R (version 2.13.0 [2011-04-13], The R Foundation for Statistical Computing, Vienna, Austria). The continuous variables are presented as medians (25th, 75th quartile range) and categorical variables in percentages. For data where the normality assumptions were suspect, the Mann-Whitney U-test was used. The chi-square test was used for comparison of categorical variables. Logistic regression was used to determine the adjusted odds ratio for type 2 diabetes by adjusting for other factors such as age, gender, family history of type 2 diabetes, alcohol consumption, serum cotinine levels, waist circumference or BMI, waist-to-hip ratio, hypertension, education status and lipid levels. Final predictors were based on backward elimination procedures. The age-standardised frequencies were calculated in 10-year intervals using the standard world population distribution as projected by the WHO for $2000-2025 .{ }^{15}$ The direct standardisation was applied. For the purpose of comparing the JIS, ATP III, and IDF definitions to identify subjects with MetS, the kappa $(k)$ statistic was calculated. Results were considered significant where $p<0.05$.

\section{Results}

A total of 642 random subjects with a mean age (standard deviation) of 50.9 (9.1) years participated. Seventy-nine (32 males and 47 females) subjects had previously diagnosed type 2 diabetes, and these subjects were not included for the prevalence of metabolic syndrome. The general characteristics and the prevalence of metabolic syndrome of the remaining 563 subjects are presented in Table 1, and data are presented as median (25th, 75th percentiles). The BMI, waist circumference, blood glucose, total cholesterol and HDL-c were significantly higher in females, while blood pressure and LDL-c were significantly higher in males.

The crude prevalence of type 2 diabetes including the previously diagnosed type 2 diabetes was 28.2\% (age-adjusted 26.3\% (95\% confidence interval (CI) $22.0-30.3$ )), and that of undiagnosed type 2 diabetes was $18.1 \%$ (age-adjusted $16.8 \%$ (13.3 - 20.4). Prediabetes (i.e. impaired fasting glucose (IFG)) was 4.4\% (age adjusted 3.2\% (1.6 4.9), impaired glucose tolerance (IGT) was 15.3\% (age adjusted 15.0\% (11.4 - 18.6)), and the combination of both IFG/IGT was $4.3 \%$ (age adjusted $3.6 \%(2.5-5.2)$ ). While prediabetes was present in subjects $<40$ years old in both sexes, type 2 diabetes was absent in males in this age group. In both sexes type 2 diabetes peaked at ages $40-49$ years, but above age 60 years, the prevalence of undiagnosed type 2 diabetes in males was 2.6 times lower than that in females (Table 2). In multivariable models, increasing age (odds ratio (OR) 1.01; 95\% CI $1.01-1.02$, per year increase in age), waist circumference (1.01 $(1.00-1.01))$, TG (1.06 (1.02 - 1.10)), and family history of diabetes $(1.17(1.09-1.26))$ were significantly associated with both diabetes mellitus and prediabetes.

Overall, there was a good agreement between the MetS criteria ( $k=0.89,95 \%$ CI 0.85 - 0.92), between JIS and IDF ( $0.97 ; 0.95$ - 0.99), JIS and ATP III $(0.86 ; 0.82-0.90)$ and IDF and ATP III $(0.83 ; 0.79$ $0.88)$. In the group of 558 subjects included in the determination of 


\section{RESEARCH}

Table 1. Characteristics of 563 participants, stratified by gender

\begin{tabular}{|c|c|c|c|}
\hline Characteristics & Male $(n=109)$ & Female $(n=454)$ & $p$-value \\
\hline Age (years) & $52(44,59)$ & $51(43,48)$ & 0.33 \\
\hline BMI $\left(\mathrm{kg} / \mathrm{m}^{2}\right)$ & $25.8(22.3,30.0)$ & $30.8(26.5,35.4)$ & $<0.0001$ \\
\hline Waist-C $(\mathrm{cm})^{*}$ & $94(81,105)$ & $98.5(88,108)$ & $<0.0001$ \\
\hline WHR & $0.93(0.87,0.98)$ & $0.87(0.82,0.92)$ & $<0.0001$ \\
\hline $\mathrm{SBP}(\mathrm{mmHg})^{*}$ & $123(114,133)$ & $118(108,130)$ & 0.013 \\
\hline $\mathrm{DBP}(\mathrm{mmHg})^{*}$ & $77(69,85)$ & $74(67,82)$ & 0.016 \\
\hline $\mathrm{FBG}(\mathrm{mmol} / \mathrm{l})$ & $5.5(5.0,7.0)$ & $5.6(5.0,6.4)$ & 0.948 \\
\hline PostBG (mmol/l) & $6.3(5.3,8.3)$ & $6.9(5.7,8.8)$ & 0.014 \\
\hline $\mathrm{HbA}_{1 \mathrm{c}}(\mathrm{mmol} / \mathrm{mol})$ & $40(37,50)$ & $41(37,45)$ & 0.2245 \\
\hline $\mathrm{HbA}_{1 \mathrm{c}}(\%)$ & $5.8(5.5,6.7)$ & $5.9(5.5,6.3)$ & 0.779 \\
\hline $\mathrm{TC}(\mathrm{mmol} / \mathrm{l})$ & $5.42(4.75,6.08)$ & $5.63(4.85,6.45)$ & 0.007 \\
\hline $\mathrm{TG}(\mathrm{mmol} / \mathrm{l})$ & $1.36(0.95,1.91)$ & $1.29(0.93,1.83)$ & 0.379 \\
\hline HDL-c (mmol/l) & $1.13(0.96,1.40)$ & $1.22(1.02,1.44)$ & 0.027 \\
\hline LDL-c $(\mathrm{mmol} / \mathrm{l})$ & $3.34(2.66,4.12)$ & $3.73(3.07,4.40)$ & 0.0004 \\
\hline Current smoking \% & 57.8 & 41.2 & 0.0017 \\
\hline Serum cotinine (ng/ml) & $24(9,325)$ & $9(9,327)$ & 0.345 \\
\hline Alcohol consumption \% & 53.2 & 22.5 & $<0.0001$ \\
\hline MetS IIS $(n=558)$ & $38(35.2 \%)$ & $308(68.4 \%)$ & $<0.0001$ \\
\hline $\operatorname{Met}^{\text {NCEP ATP III }}(n=558)$ & $29(26.8 \%)$ & $280(62.2 \%)$ & $<0.0001$ \\
\hline $\operatorname{MetS}^{\operatorname{IDF}}(n=558)$ & $33(30.6 \%)$ & $305(67.8 \%)$ & $<0.0001$ \\
\hline
\end{tabular}

Table 2. Age-specific frequency of undiagnosed type 2 diabetes according to the WHO criteria among the coloured population of South Africa. Frequencies are expressed as percentages

\begin{tabular}{|c|c|c|c|c|c|}
\hline Age groups $(n)$ & Prevalence & IFG & IGT & IFG/IGT & $\begin{array}{l}\text { Undiagnosed } \\
\text { diabetes }\end{array}$ \\
\hline \multicolumn{6}{|c|}{$30-39(12 \mathrm{M}, 65 \mathrm{~F})$} \\
\hline & Men; women & $8.3 ; 6.2$ & $16.7 ; 13.8$ & $0 ; 0$ & $0 ; 7.7$ \\
\hline & Crude, overall & 6.5 & 14.3 & 0 & 6.5 \\
\hline & Age-adjusted & $2.7(0.1-5.3)$ & $14.2(8.6-19.8)$ & 0 & $6.8(2.7-10.8)$ \\
\hline \multicolumn{6}{|c|}{$40-49(39 \mathrm{M}, 159 \mathrm{~F})$} \\
\hline & Men; women & $5.1 ; 5.0$ & $12.8 ; 11.9$ & $0 ; 4.4$ & $20.5 ; 15.7$ \\
\hline & Crude, overall & 5.1 & 12.1 & 3.5 & 14.7 \\
\hline & Age-adjusted & $4.8(1.0-8.5)$ & $11.9(6.2-17.6)$ & $3.2(0.1-6.2)$ & $15.9(9.5-22.2)$ \\
\hline \multicolumn{6}{|c|}{$50-59(41 \mathrm{M}, 137 \mathrm{~F})$} \\
\hline & Men; women & $2.4 ; 5.1$ & $12.2 ; 20.4$ & $2.4 ; 5.1$ & $17.1 ; 19.7$ \\
\hline & Crude, overall & 4.5 & 18.5 & 4.5 & 16.8 \\
\hline & Age-adjusted & $4.0(0.2-7.9)$ & $18.2(10.6-25.8)$ & $4.0(0.2-7.9)$ & $19.2(11.4-26.9)$ \\
\hline \multicolumn{6}{|l|}{$\geq 60(17 \mathrm{M}, 93 \mathrm{~F})$} \\
\hline & Men; women & $0 ; 2.2$ & $29.4 ; 14.0$ & $5.9 ; 8.6$ & $11.8 ; 31.2$ \\
\hline & Crude, overall & 1.8 & 16.4 & 8.2 & 28.2 \\
\hline & Age-adjusted & $1.7(0.6-4.0)$ & $16.7(10.0-23.3)$ & $8.3(3.4-13.3)$ & $28.3(20.3-36.4)$ \\
\hline \multicolumn{6}{|l|}{ Total 563} \\
\hline & Crude prevalence & 4.4 & 15.3 & 4.3 & 18.1 \\
\hline & Age-adjusted & $3.2(1.6-4.9)$ & $15.0(11.4-18.6)$ & $3.6(2.5-5.2)$ & $16.8(13.3-20.4)$ \\
\hline
\end{tabular}


MetS, the crude prevalence of MetS was higher with the JIS definition $(62.0 \%)$ than the IDF $(60.6 \%)$ and the NCEP ATP III (55.4\%). Irrespective of the method used, MetS was significantly more prevalent in females $(p<0.0001$ (Table 1$))$. Central obesity was the most common abnormal criterion, followed by raised blood pressure, reduced HDL-c, hyperglycaemia and raised TG. MetS increased with age; $81.2 \%$ of subjects with undiagnosed type 2 diabetes, and $83.7 \%$ with non-diabetic hyperglycaemia, had MetS, compared with $46.9 \%$ normoglycaemic subjects using the JIS criteria.

\section{Discussion}

We provide evidence that the mixed ancestry population of the Western Cape is experiencing the predicted diabetes epidemic in developing countries. In contrast to the last report published 12 years ago in a peri-urban coloured population of the Western Cape, ${ }^{2}$ we found a high prevalence (28.2\%) of type 2 diabetes, which included 80 previously diagnosed type 2 diabetes and 101 undiagnosed type 2 diabetes subjects. The age-adjusted prevalence of type 2 diabetes (26.3\%) was very high compared with the 2010 estimates for adults aged 20 - 79 years from South Africa, but similar to Nauru which has one of the highest prevalence rates in the world. ${ }^{16}$ The sharp increase in the prevalence of type 2 diabetes between these studies may be due to the geographical location of these populations, significant differences in obesity, their economic transition, and the different criteria used for diagnosing diabetes. The limited studies in South Africa have shown a positive rural-to-urban gradient in terms of the prevalence of type 2 diabetes, ${ }^{2-4,12}$ and the sample for this study was taken from an urban coloured population located approximately $50 \mathrm{~km}$ from the previous study. ${ }^{2}$ Furthermore, our study subjects were more obese, with larger waist circumferences. The mean waist circumference was $98.5 \mathrm{~cm}$ in females and $94.0 \mathrm{~cm}$ in males, resulting in $87.9 \%$ and $42.2 \%$ central obesity rates as defined by the IDF criteria, respectively. Waist circumference together with family history of type 2 diabetes, TG and increasing age, were significantly associated with the development of diabetes mellitus.

Despite the availability of primary healthcare, 101 (55.8\%) out of 181 subjects with type 2 diabetes were not aware of their condition. These findings suggest that the primary healthcare system may be inadequate to identify type 2 diabetes cases and those at high risk of type 2 diabetes. Individuals with undiagnosed type 2 diabetes or pre-diabetes exhibit a high prevalence of MetS and are therefore at a higher future risk of CVD. Though the prevalence of MetS was lower when using the ATP III definition than the IDF and the JIS, there was generally good concordance between all three MetS criteria. To our knowledge, this is the first population-based report on the prevalence of MetS in the coloured population and is the highest observed among sub-Saharan populations. ${ }^{8,17}$ The JIS definition for identifying subjects with MetS has been used in very few studies from Africa. ${ }^{17}$ Compared with other sub-Saharan regions that used IDF or ATP III, ${ }^{8}$ our study prevalence of MetS was much higher, probably reflecting the high diabetes prevalence in our population. Irrespective of the MetS definition used, we observed MetS to be significantly more prevalent in females, a difference unquestionably due to the increased central obesity in females.
There are a few limitations to this study. On account of logistic constraints, blood glucose measurements were not repeated in asymptomatic subjects who were found to have pre-diabetes or type 2 diabetes, as has been previously suggested. ${ }^{14}$ The nature of this study is cross-sectional, with high female-to-male participation, which is a common trend in South African population studies.

In conclusion: our data are consistent with literature demonstrating a strong association between MetS and type 2 diabetes. Contrary to previous reports, the coloured population of South Africa has a high CVD risk, as shown by the high prevalence of type 2 diabetes accompanied by MetS. This finding emphasises the need for South Africa to reorganise its primary healthcare system to manage chronic lifestyle diseases and curb this new epidemic alongside the existing infectious disease burden.

Acknowledgements. We thank the Bellville South community. This research was supported by a grant from the University Research Fund of the Cape Peninsula University of Technology, Cape Town.

\section{References}

1. de Wit E, Delport W, Rugamika CE, et al. Genome-wide analysis of the structure of the South African coloured population in the Western Cape. Hum Genet 2010;128:145-153. [http://dx.doi.org/10.1007/ s00439-010-0836-1]

2. Levitt NS, Steyn K, Lambert EV, et al. Modifiable risk factors for Type 2 diabetes mellitus in a periurban community in South Africa. Diabet Med 1999;16:946-950. [http://dx.doi.org/10.1046/.14645491.1999.00185.x]

3. Erasmus RT, Blanco-Blanco E, Okesina AB, Matsha T, Gqweta Z, Mesa JA. Prevalence of diabetes mellitus and impaired glucose tolerance in factory workers from Transkei, South Africa. S Afr Med J 2001:91:157-160.

4. Motala AA, Pirie FJ, Gouws E, Amod A, Omar MA. High incidence of type 2 diabetes mellitus in South African Indians: a 10-year follow-up study. Diabet Med 2003;20:23-30. [http://dx.doi.org/10.1046/ A.1464-5491.2003.00782 x]

5. Metascreen Writing Committee, Bonadonna RC, Cucinotta D, Fedele D, Riccardi G, Tiengo A. The Metascreen Writing Committee, Bonadonna RC, Cucinotta D, Fedele D, Riccardi G, Tiengo A. The
metabolic syndrome is a risk indicator of microvascular and macrovascular complications in diabetes: metabolic syndrome is a risk indicator of microvascular and macrovascular complications in diabetes:
results from Metascreen, a multicenter diabetes clinic-based survey. Diabetes Care 2006;29:2701-2707. 6.

6. Matsha TE, Hassan MS, Kidd M, Erasmus RT. The 30-year cardiovascular risk profile of South African 6. Matsha TE, Hassan MS, Kidd M, Erasmus RT. The 30-year cardiovascular risk profile of South Africans
with diagnosed diabetes, undiagnosed diabetes, pre-diabetes or normoglycaemia: The Bellville-South with diagnosed diabetes, undiagnosed diabetes, pre-diabetes or normoglycaemia: The Bellville-Soll

Ntyintyane LM, Panz VR, Raal FJ, Gill GV. Metabolic syndrome, undiagnosed diabetes mellitus and Ntyintyane LM, Panz VR, Raal F, Gill GV. Metabolic syndrome, undiagnosed diabetes mellitus and
insulin resistance are highly prevalent in urbanised South African blacks with coronary artery disease. Cardiovasc J S Afr 2006;17:50-55.

8. Kengne AP, Limen SN, Sobngwi E, Djouogo CF, Nouedoui C. Metabolic syndrome in type 2 diabetes: comparative prevalence according to two sets of diagnostic criteria in sub-Saharan Africans. Diabeto

9. Alberti KG, Eckel RH, Grundy SM, et al. Harmonizing the Metabolic Syndrome: A Joint Interim Statement of the International Diabetes Federation Task Force on Epidemiology and Prevention National Heart, Lung, and Blood Institute; American Heart Association; World Heart Federation; International Atherosclerosis Society; and International Association for the Study of Obesi Circulation 2009;120:1640-1645. [http://dx.doi.org/10.1161/CIRCULATIONAHA.109.192644]

10. Zemlin AE, Matsha TE, Hassan MS, Erasmus RT. HbAlc of $6.5 \%$ to diagnose diabetes mellitus - does it work for us? - The Bellville South Africa Study. PLoS ONE 2011;6:e22558. [http://dx.doi. org/10.1371/journal.pone.0022558

11. Ewing JA. Detecting alcoholism: The CAGE Questionnaire. JAMA 1984;252:1905-1907.

12. Bradshaw D, Bourne D, Schneider M, Sayed R. Mortality Patterns of Chronic Diseases of Lifestyle in South Africa. In: Fourie J, Steyn K, eds. Chronic Diseases of Lifestyle in South Africa. Tygerberg: Medical Research Council (MRC) Technical Report, 1995: 5-31

13. World Health Organization International Society of Hypertension Guidelines for the Management of Hypertension. J Hypertens 1999;17:151-183.

14. Alberti KG, Zimmet PZ. Definition, diagnosis and classification of diabetes mellitus and its complications. Part 1: diagnosis and classification of diabetes mellitus provisional report of a WHO consultation. Diabet Med 1998;15:539-553.

15. Ahmad OB, Boschi-Pinto C, Lopez AD, Murray CJL, Lozano R, Inoue M. Age standardization of rates. A new WHO standard. EIP/GPE/EBD. GPE Discussion Paper Series. WHO 2001;31:1-14

16. Shaw JE, Sicree RA, Zimmet PZ. Global estimates of the prevalence of diabetes for 2010 and 2030 Diabetes Res Clin Pract 2010;87:4-14. [http://dx.doi.org/10.1016/j.diabres.2009.10.007]

17. Motala AA, Esterhuizen T, Pirie FJ, Omar MA. The prevalence of metabolic syndrome and determination of the optimal waist circumference cutoff points in a rural South African community. Diabetes Care 2011;34:1032-1037. [http://dx.doi.org/10.2337/dc10-1921] 